\title{
Reflexões acerca dos estilos de aprendizagem no contexto do Ensino Fundamental
}

\author{
Reflections on learning styles in the context of Elementary School \\ Reflexiones sobre estilos de aprendizaje en el contexto de la Escuela Primaria
}

Recebido: 12/09/2021 | Revisado: 17/09/2021 | Aceito: 18/09/2021 | Publicado: 19/09/2021

\author{
Rhenan Ferraz de Jesus \\ ORCID: https://orcid.org/0000-0002-1717-6593 \\ Instituto Federal de Educação, Ciência e Tecnologia Farroupilha, Brasil \\ E-mail: rhenan.jesus@iffarroupilha.edu.br \\ Vanderlei Folmer \\ ORCID: https://orcid.org/0000-0001-6940-9080 \\ Universidade Federal do Pampa, Brasil \\ E-mail: vanderleifolmer@unipampa.edu.br
}

\begin{abstract}
Resumo
Analisar as preferências de estilo de aprendizagem de estudantes e professores do Ensino Fundamental. Trata-se de uma pesquisa qualitativa do tipo estudo exploratório. Participaram efetivamente do estudo 193 estudantes e 19 professores do Ensino Fundamental, pertencentes a duas escolas da rede pública de ensino de Alegrete-RS. Foi utilizado o Inventário dos Estilos de Aprendizagem - IEA (Learning Style Inventory - LSI) de David Kolb para a coleta dos dados. Observou-se que os estilos de aprendizagem predominantes no perfil dos estudantes foram o Assimilador $(n=89 ; 46,25 \%)$ e o Convergente $(n=61 ; 31,73 \%)$. Notou-se que o estilo de aprendizagem Convergente predomina o perfil dos professores $(n=9 ; 47,37 \%)$ respondentes. Percebeu que há uma distribuição mais uniforme entre os estilos de aprendizagem dos professores do que os estilos dos estudantes. Isso leva a entender que os estilos de ensino predominante dos professores apresentam compatibilidade com os estilos de aprendizagem dos estudantes. Notou-se que há vários perfis de aprendizado e de ensino. Comprendeu-se que os estilos de aprendizagem dos professores, que se baseiam para ensinar, abarcam diversos estilos de aprendizagem dos seus alunos. A partir disso, entende-se que o perfil dos professores possibilita explorar as distintas maneiras que os estudantes tendem a aprender. Acredita-se que identificar os estilos de aprendizagem é uma estratégia pedagógica que, aliada ao uso de metodologias inovadoras no ensino, tem potencial de auxiliar na eficácia de intervenções educativas e na qualidade do processo de ensino e de aprendizagem.
\end{abstract}

Palavras-chave: Ensino; Aprendizagem; Educação Básica.

\begin{abstract}
Analyze the learning style preferences of elementary school students and teachers. This is a qualitative research of the exploratory study type. 193 students and 19 elementary school teachers from two public schools in Alegrete-RS effectively participated in the study. The David Kolb Learning Styles Inventory (LSI) was used for data collection. It was observed that the predominant learning styles in the profile of the students were Assimilating $(n=89 ; 46,25 \%)$ and Convergent $(n=61 ; 31,73 \%)$. It was noted that the Convergent learning style predominates the profile of teachers $(n=9 ; 47,37 \%)$ respondents. It noticed that there is a more even distribution between the learning styles of teachers than the styles of students. It is understood that the predominant teaching styles of teachers are compatible with students' learning styles. It was noted that there are several learning and teaching profiles. It was understood that the learning styles of teachers, which they are based on to teach, encompass different learning styles of their students. From this, it is understood that the profile of teachers makes it possible to explore the different ways that students tend to learn. It is believed that identifying learning styles is a pedagogical strategy that, combined with the use of innovative methodologies on teaching, has the potential to help in the effectiveness of educational interventions. Including the quality of the teaching and learning process.
\end{abstract}

Keywords: Teaching; Learning; Basic education.

\section{Resumen}

Analizar las preferencias de estilo de aprendizaje de los estudiantes y maestros de la escuela primaria. Se trata de una investigación cualitativa y un estudio exploratorio. En el estudio participaron efectivamente 193 estudiantes y 19 maestros de primaria de dos escuelas públicas de Alegrete-RS. Se utilizó el Inventario de Estilos de Aprendizaje IEA (Learning Style Inventory - LSI) de David Kolb para recopilar datos de la investigación. Se observó que los estilos de aprendizaje predominantes en el perfil de los estudiantes fueron Asimilante $(\mathrm{n}=89 ; 46,25 \%)$ y Convergente $(n=61 ; 31,73 \%)$. Se observó que el estilo de aprendizaje Convergente predomina en el perfil de los docentes $(n=9 ;$ 47,37\%) encuestados. Observó que hay una distribución más uniforme entre los estilos de aprendizaje de los profesores que entre los estilos de los estudiantes. Esto lleva a entender que los estilos de enseñanza predominantes de 
los docentes son compatibles con los estilos de aprendizaje de los estudiantes. Se señaló que existen varios perfiles de aprendizaje y enseñanza. Se entendió que los estilos de aprendizaje de los docentes, en los que se basan para enseñar, abarcan diferentes estilos de aprendizaje de sus alumnos. A partir de esto, se entiende que el perfil de los docentes permite explorar las diferentes formas en que los estudiantes tienden a aprender. Se cree que la identificación de estilos de aprendizaje es una estrategia pedagógica que, combinada con el uso de metodologías de enseñanza innovadoras, tiene el potencial de ayudar en la efectividad de las intervenciones educativas y en la calidad del proceso de enseñanza y aprendizaje.

Palabras clave: Enseñanza; Aprendizaje; Educación básica.

\section{Introdução}

Há muito tempo a escola é considerada um espaço primordial para se ensinar e aprender. Sabe-se que o ensino tradicional tem sido enfatizado nas escolas. Este ensino ora centraliza-se no conhecimento e o professor como detentor desse saber, ora no método de ensino, o qual é característico pela transmissão de informações sobre algo a título de conhecimento dos educandos.

Em decorrência desse modo de ensinar, a aprendizagem comumente tem sido conhecida pelas seguintes características: a) memorização - retenção da informação; b) repetição sistemática de conteúdos escolares; e c) resolução de uma longa lista de exercícios. Um dos elementos determinantes no processo de ensino e de aprendizagem é a didática utilizada em aula pelos professores. Esta pode influenciar no aprendizado dos estudantes (Assunção \& Nascimento, 2019).

No cenário atual da educação, é preocupante os níveis de aprendizagem dos educandos durante a escolarização. Segundo os resultados da avaliação do Sistema de Avaliação da Educação Básica - Saeb (Brasil, 2018), nos Anos Finais (6º ao $9^{\circ}$ ) do Ensino Fundamental, houve uma pequena melhora quanto aos níveis de aprendizagem dos alunos de 2015 para 2017 em Escalas de Proficiência de Língua Portuguesa e de Matemática, embora os atuais níveis apresentados (nível 3 de proficiência média) sejam considerados como insuficientes pelo Ministério da Educação - MEC (Brasil, 2018). Essa informação demonstra que, nos Anos Finais do Ensino Fundamental, a aprendizagem em Língua Portuguesa e Matemática estão estagnados.

É possível que alguns aspectos importantes não estejam sendo levados em conta para a realização dessa avaliação. Exemplo disso é a falta de compreensão dos termos científicos por parte dos estudantes e a falta em estabelecer uma ligação entre o conhecimento ensinado e o cotidiano dos alunos durante as aulas de Ciências (Paim, et al., 2021), a realidade que os professores se deparam pela falta de recursos e espaço para desenvolvimento de atividades extracurriculares e o desinteresse dos alunos (Prandina \& Santos, 2017). Esses aspectos corroboram para que muitos alunos apresentem dificuldades durante o processo de aprendizagem, inclusive, eles colaboram para que sejam apresentados resultados não suficientes, na visão do MEC.

Tão logo, questiona-se: enquanto se ensina, os estudantes aprendem? Pode ser que haja situações em que os estudantes não "aprendam" ou não consigam processar as informações ensinadas. Da mesma forma que ruídos ou falhas no processo de comunicação entre professor e estudante ocorram, ou até mesmo haver lacunas na transposição didática, implicando na aprendizagem. O fato é que há de se considerar que várias formas de ensinar e de aprender existam. A partir disso, como problema de pesquisa, questiona-se se os diferentes modos de aprender dos alunos são contemplados a partir dos estilos de ensinar dos professores.

Diante da gama de concepções sobre aprendizagem, surgem alguns modelos de aprendizagem que levam em conta a existência de distintas formas de aprender entre os indivíduos, bem como o conhecimento sobre as potencialidades e fragilidades dos aprendentes (Leite, et al., 2020). O Inventário dos Estilos de Aprendizagem - IEA (Learning Style Inventory LSI) tem sido utilizado na Educação Básica para identificar as diferentes maneiras que os estudantes aprendem. Este instrumento é utilizado por Kolb $(1984 ; 2015)$ para identificar o perfil do sujeito alvo da aprendizagem.

Kolb (1984, p. 24) define estilo de aprendizagem como "um estado duradouro e estável que deriva de configurações 
consistentes das transações entre o indivíduo e o seu meio ambiente". Estilo de Aprendizagem pode ser entendido como um método que a pessoa usa para adquirir conhecimento, sendo que cada indivíduo aprende do seu modo pessoal e único (Cerqueira, 2000; 2008). Kolb (1984; 2015) identificou quatro estilos de aprendizagem. O Quadro 1 apresenta características em cada um desses estilos.

Quadro 1. Características dos estilos de aprendizagem.

\begin{tabular}{|c|c|}
\hline $\begin{array}{l}\text { Estilo de } \\
\text { aprendizagem }\end{array}$ & Características \\
\hline Divergente & $\begin{array}{l}\text { - aprende melhor combinando sensações com observações, ou seja através de atividades práticas seguidas de um } \\
\text { retorno; } \\
\text { - possui muita sensibilidade artística e conseguem ver as coisas de perspectivas diferentes; } \\
\text { - prefere observar ao invés de agir; } \\
\text { - suas estratégias para a solução de problemas iniciam coletando informações para em seguida usarem a criatividade e } \\
\text { a inventividade para oferecer mais de uma solução possível; } \\
\text { - a denominação "divergentes" se dá pelo fato de terem bom desempenho em situações que requerem geração de } \\
\text { ideias, como grupos de trabalho e brainstorms (tempestade de ideias); } \\
\text { - possuem vasto interesse cultural e gostam de pessoas; } \\
\text { - preferem trabalhar em grupo, ouvindo sugestões com mente aberta e recebendo feedbacks pessoais; } \\
\text { - gostam de autonomia na busca de conhecimento; } \\
\text { - esse estilo também é conhecido como REFLEXIVO }\end{array}$ \\
\hline Assin & $\begin{array}{l}\text { - aprende melhor combinando observação e pensamento, por isso suas preferências por palestras, conferências e aulas; } \\
\text { - para eles, ideias e conceitos abstratos são mais importantes do que pessoas e pode ser percebido como pouco } \\
\text { sociável; } \\
\text { - tem facilidade com números e modelos conceituais, preferindo especulações abstratas em detrimento de situações } \\
\text { práticas; } \\
\text { - compreende as informações de forma ampla e as organizam de forma clara e lógica; } \\
\text { - tem propensão para a carreira científica; } \\
\text { - gosta de explorar modelos analíticos e de ter tempo para pensar e refletir sobre as coisas; } \\
\text { - esse estilo também é conhecido como TEÓRICO. }\end{array}$ \\
\hline Convergente & $\begin{array}{l}\text { - aprende melhor pensando e realizando; } \\
\text { - combina o gosto de colocar "a mão na massa" com aspectos teóricos; } \\
\text { - gosta de realizar atividades com indicações sequenciais detalhadas (como aquelas dos manuais de operação de } \\
\text { aparelhos), solucionar problemas específicos e testar hipóteses (tentativa e erro); } \\
\text { - tem habilidades em encontrar aplicações práticas para ideias e teorias; } \\
\text { - pessoas desse estilo possuem poucas habilidades sociais e intrapessoais, preferindo ambientes de aprendizagem mais } \\
\text { tranquilos (ex.: gosta de trabalha sozinho realizando tarefas técnicas sem se relacionarem com outras pessoas); } \\
\text { - parecem se sair melhor em situações nas quais existe uma única resposta ou solução correta para cada pergunta ou } \\
\text { problema; } \\
\text { - não tem dificuldades ao experimentar inovações para solucionar problemas práticos } \\
\text { - esse estilo também é conhecido como PRAGMÁTICO. }\end{array}$ \\
\hline Acomodador & $\begin{array}{l}\text { - aprende melhor experimentando e realizando, como, por exemplo, através de atividades práticas, apresentações, role- } \\
\text { plays e debates; } \\
\text { - combina o gosto de colocar "a mão na massa" com atividades concretas; } \\
\text { - tem capacidade de se sobressair e acomodar ou adaptar a circunstâncias imediatas específicas; } \\
\text { - utiliza mais a intuição do que a lógica e tem a tendência a se arriscar mais a ousar mais; } \\
\text { - costuma utilizar a opinião de outras pessoas ao invés das suas próprias, por isso geralmente faz muitas perguntas; } \\
\text { - assume uma abordagem prática e vivencial; } \\
\text { - é sociável e gosta de trabalhar em equipe; } \\
\text { - geralmente exerce um papel importante em situações onde são necessárias ações e iniciativas para a realização de } \\
\text { tarefas; } \\
\text { - por terem pouca habilidade analítica são impulsivas e, as vezes, é percebido como impaciente e pressionador; } \\
\text { - esse estilo também é conhecido como ATIVISTA. }\end{array}$ \\
\hline
\end{tabular}

Fonte: Adaptado a partir de Kolb (1984; 2015). 
Acrani et al. (2020) citam a utilização do inventário de Kolb (1984; 2015), para identificar as preferências de aprendizagem de alunos do final do Ensino Fundamental, trata-se de uma útil ferramenta de investigação que serviu para dar sustentação a uma série de intervenções pedagógicas, favorecendo a escolha de metodologias ativas. Jesus et al. (2017) afirmam que investigar o estilo de aprendizagem dos estudantes pode contribuir para uma compreensão acerca do ato de aprender, o que trará implicações, também, na ação de ensinar, contribuindo para promover uma relação dialética no ensino.

Como propalam estes mesmos autores, conhecer os estilos de aprendizagem do discente pode ser um diferencial nas ações do docente durante o processo de ensino e de aprendizagem (Jesus, et al., 2017). Pois, o professor tem a possibilidade, em sala de aula, de utilizar distintas estratégias e metodologias de ensino que possam contemplar diversas preferências e estilos de aprendizagem, beneficiando aos alunos ao considerar suas diferenças nas formas de aprender (Acrani, et al., 2020).

Sendo assim, identificar as diferentes maneiras de aprender dos estudantes e os distintos modos de aprender, que os professores se baseiam para ensinar, pulsa como uma possibilidade. Em especial, para pensar se, ao averiguar os estilos de aprendizagem, essa ação pode ser encarada como uma estratégia pedagógica que tenha potencial de facilitar a produção de intervenções mais efetivas nas práticas escolares, ao exemplo do uso de metodologias ativas.

Segundo o que relatam Freeman et al. (2014), em sua análise realizada em 225 estudos, há eficácia no uso de Metodologias ativas ao se comparar diferentes metodologias de ensino (tradicional $\mathrm{x}$ aprendizagem ativa), quando se busca explorar distintas maneiras que os estudantes aprendem. $\mathrm{O}$ trabalho destes mesmos autores observou que alunos, que cursavam disciplinas oferecidas com aprendizado ativo, obtinham notas ligeiramente mais altas e uma menor taxa de reprovação (Freeman, et al., 2014). Para as abordagens ativas, foram utilizadas diferentes estratégias como a resolução de problemas em grupo, a elaboração de planilhas, a experimentação em laboratório, etc..

Diante do que foi exposto, o objetivo deste estudo foi analisar as preferências de estilo de aprendizagem de estudantes e professores do Ensino Fundamental, em escolas da rede pública de ensino em Alegrete-RS.

\section{Metodologia}

O presente trabalho é de natureza qualitativa, onde se busca analisar as preferências dos estilos de aprendizagem de estudantes e professores do Ensino Fundamental. Na pesquisa qualitativa, entende-se que os questionamentos dos fenômenos e sujeitos investigados irão possibilitar perceber aquilo que eles experimentam em suas instituições, o modo como interpretam as experiências e como estruturam o mundo social em que vivem (Bogdan \& Biklen, 1994), deste modo, corroborando para atingir o objetivo do estudo. Trata-se de uma pesquisa exploratória (Gil, 2008, p. 27), a qual tem "como principal finalidade desenvolver, esclarecer e modificar conceitos e ideias, tendo em vista a formulação de problemas mais precisos ou hipóteses pesquisáveis para estudos posteriores".

De modo a complementar a pesquisa, utilizou-se quantificações para analisar frequências, o que permitiu a realização de análises numéricas de dados, possibilitando estabelecer causas e relações (Minayo \& Guerriero, 2014). A quantificação não descaracteriza a natureza qualitativa do processo investigativo, pois, “[...] as questões a investigar não se estabelecem mediante a operacionalização de variáveis, sendo, outrossim, formuladas com o objetivo de investigar os fenômenos em toda a sua complexidade e em contexto natural" (Bogdan \& Biklen, 1994, p. 16).

Respeitando os critérios éticos da pesquisa, conforme os termos da Resolução 466/2012 e 510/2016 do Conselho Nacional de Saúde, este estudo foi aprovado pelo Comitê de Ética em Pesquisa da Universidade Federal do Rio Grande do Sul, sob Parecer no. 3.720.486, de 22 de novembro de 2019. A Secretaria de Educação, Cultura, Esporte e Lazer (SECEL), as escolas (equipes diretivas), os professores e os estudantes, juntamente com seus pais e/ou responsáveis, assinaram, respectivamente, uma Carta de Anuência, um Termo de Autorização Institucional, um Termo de Consentimento Livre e 
Esclarecido, um Termo de Assentimento Esclarecido (estudantes) e um Termo de Consentimento Livre e Esclarecido (pais e/ou responsáveis dos estudantes).

O sigilo das informações prestadas e o anonimato dos partícipes foi mantido, utilizando pseudônimos aos professores (P1, P2, P3 e assim por diante) e aos alunos (A1, A2, A3 e assim por diante). Participaram do presente estudo 19 professores de distintas áreas do conhecimento e 193 estudantes (elegíveis) ${ }^{1}$ do $5^{\circ}, 6^{\circ}, 7^{\circ}$ e $8^{\circ}$ anos ${ }^{2}$ do Ensino Fundamental, de duas escolas da rede pública de ensino do município de Alegrete-RS. O instrumento usado para coletar os dados da pesquisa foi o IEA de Kolb (1984; 2015), como mostra a Figura 1.

Figura 1. Inventário dos Estilo de Aprendizagem, aplicado aos estudantes e aos professores.

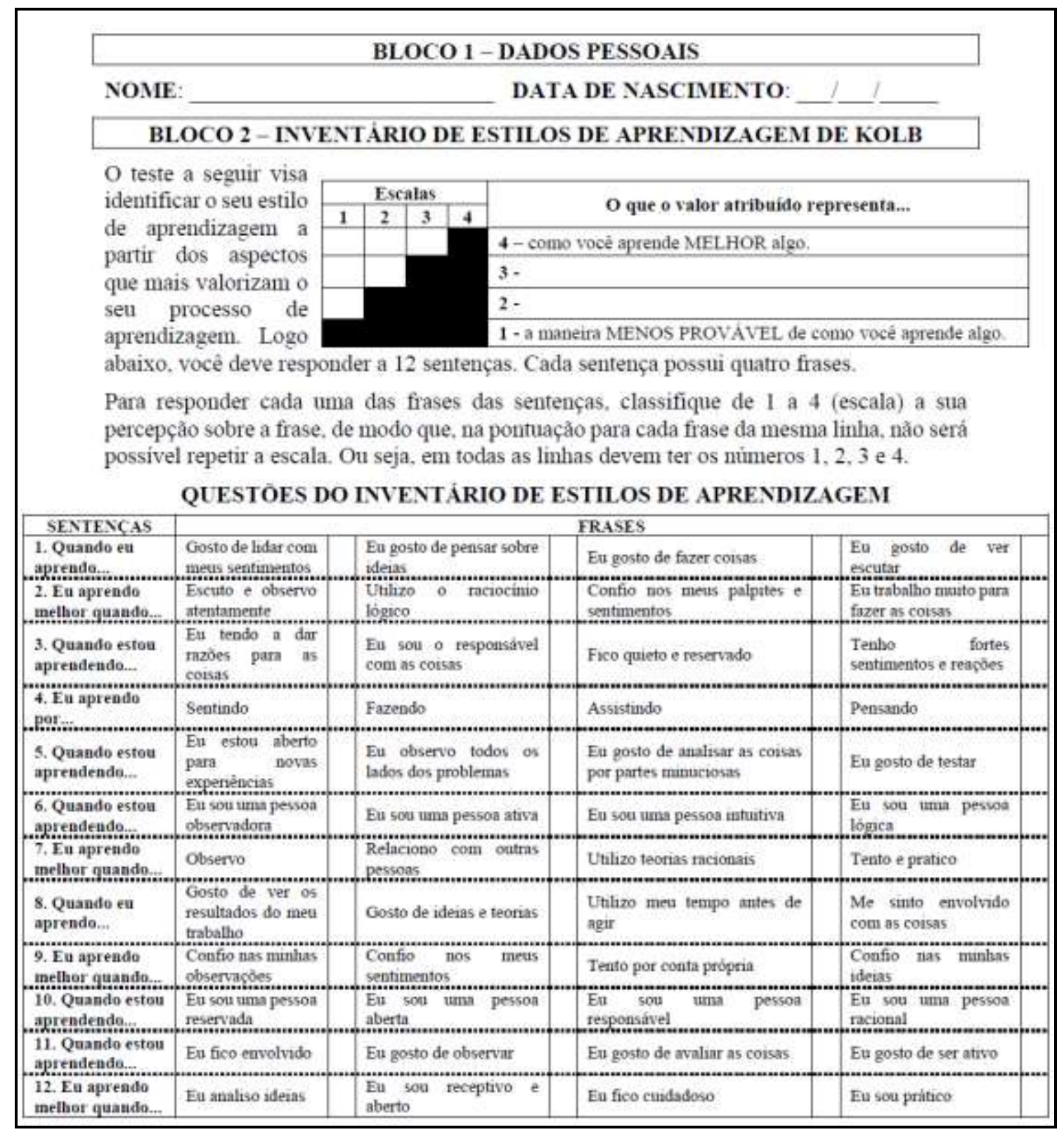

Fonte: Kolb (2015).

\footnotetext{
${ }^{1}$ O número total de estudantes que participaram do estudo foi 197 estudantes. Invalidou-se quatro questionários respondidos, pois, haviam questões que não foram preenchidas adequadamente pelos estudantes.

${ }^{2}$ Foi estabelecido um critério para a participação desses estudantes. Devido a este estudo ser um diagnóstico inicial, delimitou-se a participação apenas de alunos que seriam, supostamente, pertencentes aos próximos anos escolares, ou seja, o público-alvo seria estudantes dos Anos Finais do Ensino Fundamental $\left(6^{\circ}\right.$ ao $\left.9^{\circ}\right)$. Estes seriam convidados a participarem de um novo estudo no próximo ano e submetidos a nova avaliação para averiguar se os estilos de aprendizagem dos estudantes apresentariam modificações após a oferta de um curso de formação para os professores destes estudantes.
} 
O teste do IEA foi aplicado em uma sala de aula individualmente, com cada aluno, disponibilizado por cada uma das escolas participantes, o que ajudou a evitar possíveis interferências no momento de responder as questões perquiridas. O professor regente de sala de aula contribuiu no processo de coleta de dados, deixando um tempo de sua aula para que os estudantes fossem respondendo as questões na sala reservada para tal. À medida que os partícipes iam apresentando dúvidas, o pesquisador ia auxiliando, sem induzir nas suas respostas, ficando a cargo da sua interpretação. Os professores também responderam o IEA na mesma sala que os estudantes. O período da realização da coleta de dados compreendeu entre os meses de outubro e novembro de 2019.

Para realizar a análise dos dados coletados no IEA, um dos pesquisadores utilizou um link, ${ }^{3}$ que foi construído pelo Centro de Ciências Humanas, Letras e Artes da Universidade Federal da Paraíba. O referido endereço eletrônico dispõe o mesmo tipo de IEA aplicado neste estudo para identificar os estilos de aprendizagem, com base em Kolb (2015). De acordo com as respostas dadas pelos participantes da pesquisa, esse pesquisador ia repetindo as mesmas alternativas para gerar o resultado da análise dos dados. A Figura 2 exemplifica o acesso ao sítio eletrônico (A), a simulação ao preenchimento das alternativas conforme a resposta de cada participante (B), e o momento em que os resultados das preferências de aprendizagens iam sendo geradas (C), para posterior catalogação dos dados analisados em um arquivo de planilhas de Excel. De posse dessas informações, a partir dos dados da planilha foram gerados gráficos para melhor ilustrar os achados da pesquisa.

Figura 2. Procedimento realizado para analisar as respostas dadas ao IEA.

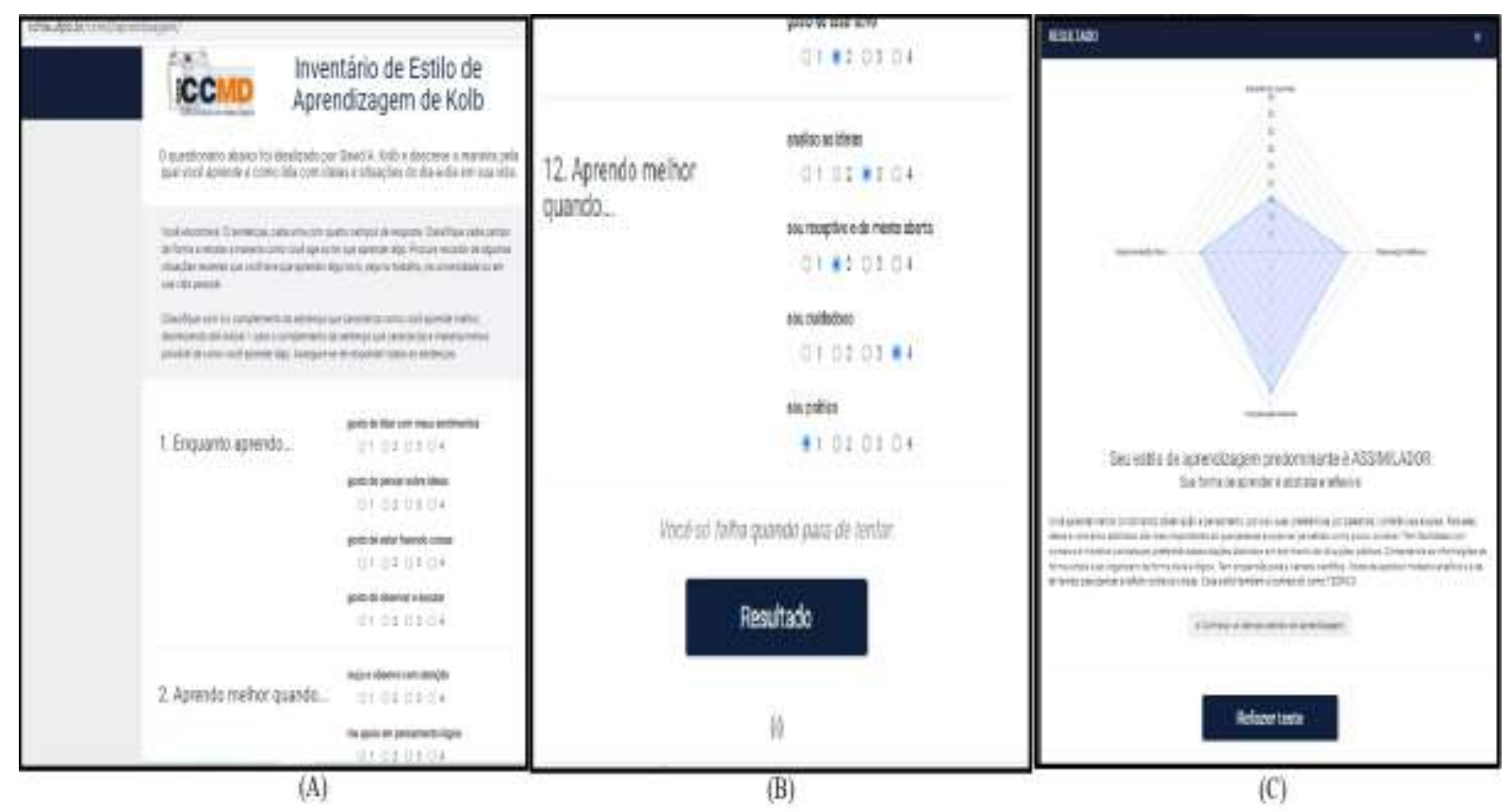

Fonte: Autores (2021).

\section{Resultados e Discussão}

Os estudantes $(\mathrm{n}=193)$ apresentaram idade média de 12,42 (dp $\pm 1,42)$ anos, na faixa etária de 9 a 16 anos para ambos os sexos, sendo desses 52,33\% ( $n=101)$ meninas e 47,67\% ( $n=92)$ meninos. De acordo com a Figura 3, percebeu-se que os estilos de aprendizagem que prevalecem no perfil dos estudantes do Ensino Fundamental foram o Assimilador, com 46,25\% $(\mathrm{n}=89)$, e o Convergente, representando $31,73 \%(\mathrm{n}=61)$ dos respondentes.

${ }^{3}$ Recuperado de: http://www.cchla.ufpb.br/ccmd/aprendizagem/. 
Figura 3. Estilo de aprendizagem dos estudantes.

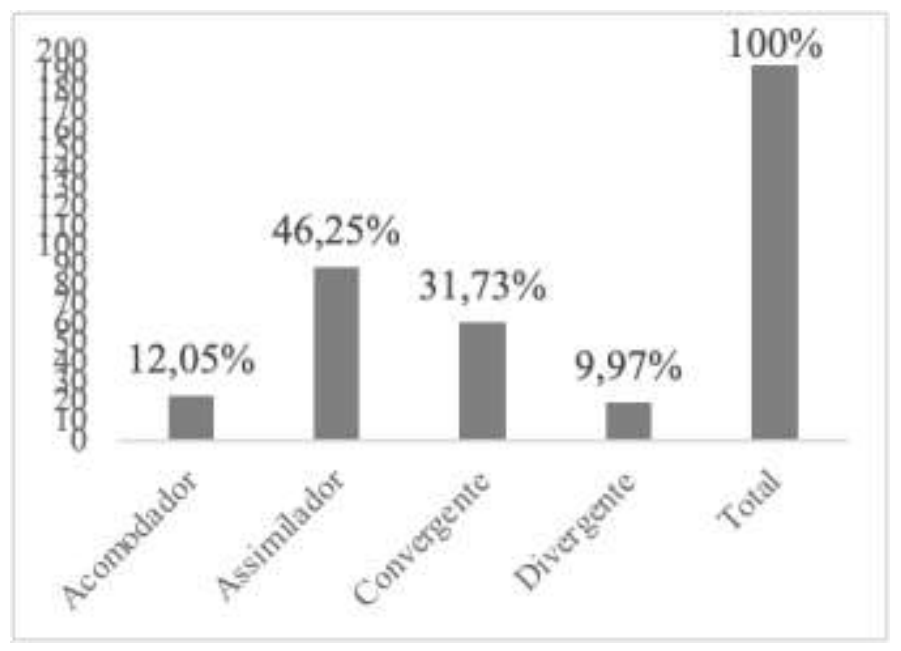

Fonte: Autores (2021).

Os achados dialogam com os estudos realizados por Nogueira et al. (2012), Souza et al. (2013) e Lima Filho et al. (2016), onde o estilo de aprendizagem predominante em estudantes foi o Assimilador. A pesquisa de Nogueira et al. (2012), onde participaram 109 alunos de um curso à distância, evidenciou que a maior parte dos discentes pertencia ao estilo Assimilador. A investigação de Souza et al. (2013) mostrou que o estilo de aprendizagem com maior escore foi o Assimilador, com 46,2\% da amostra. Lima Filho et al. (2016) realizaram um estudo com 459 acadêmicos do curso de Ciências Contábeis com o propósito de identificar o estilo de aprendizagem predominante. Igualmente, os achados mostraram haver predominância do estilo de aprendizagem Assimilador.

O estilo de aprendizagem Assimilador é característico em pessoas que combinam a observação e o pensamento para aprender, onde suas preferências são palestras, conferências e aulas. As pessoas com este estilo têm mais facilidade para reter o conhecimento a partir das ideias e conceitos abstratos, usando a lógica e explorando modelos analíticos (Kolb, 2015). Os estudantes com esse estilo de aprendizagem, comumente, aprendem melhor a partir da abordagem de um professor expositor, que utiliza métodos tradicionais para ensinar, a exemplo de aulas expositivas (Souza et al., 2013).

Já em estudo de Acrani et al. (2020), onde participaram 46 estudantes do $8^{\circ}$ e $9^{\circ}$ anos do Ensino Fundamental, entre 13 a 16 anos, os resultados observados apontam que a maioria dos investigados, aproximadamente $46 \%$ do total de alunos, foi classificado no estilo de aprendizagem Convergente. Os alunos participantes deste estudo externaram que a forma preferida de seu aprendizado envolve a aula tradicional, onde escutam o professor, bem como destacaram que também gostam da utilização de esquemas e exercícios para melhor aprender (Acrani, et al., 2020). Em estudo de Santos et al. (2018), o estilo de aprendizagem predominante dos alunos foi o Convergente. Conforme esse achado, 48,3\% (n=73) dos pesquisados possuem um estilo de aprendizagem em que os acadêmicos acabam se destacando ao utilizar na prática as ideias e teorias, onde eles preferem se envolver em situações ou problemas técnicos, a temas sociais e interpessoais (Santos, et al., 2018).

O que se percebe, assim como resultados trazidos no presente trabalho, é que os estudantes do $5^{\circ}, 6^{\circ}, 7^{\circ}$ e $8^{\circ}$ anos do Ensino Fundamental, também apresentam o estilo de aprendizagem Convergente (Figura 3) como preferência para melhor aprender, seja pensando, escutando ou vendo, combinando os aspectos teóricos e práticos (Kolb, 2015). O estilo de aprendizagem Convergente se refere, de certo modo, àquelas pessoas que buscam aplicar na prática as ideias. As pessoas que tendem a aprender por este estilo gostam de resolver problemas e tomar decisões (Cerqueira, 2000; 2008). Exemplos de atividades que essa preferência de aprendizagem envolve são aulas de simulações e experiências em laboratório. 
Os professores $(\mathrm{n}=19)$ apresentaram idade média de $42(\mathrm{dp} \pm 8,41)$ anos, na faixa etária de 30 a 60 anos para ambos os sexos, sendo desses 78,95\% ( $n=14)$ mulheres e 21,05\% $(n=4)$ homens. A partir da Figura 4, foi possível notar que o estilo de aprendizagem Convergente predomina no perfil dos professores, com 47,37\% (n=9) dos respondentes. Percebe-se também que há uma distribuição mais uniforme entre os estilos de aprendizagem dos professores do que os estilos dos estudantes.

Figura 4. Estilo de aprendizagem que os professores se baseiam para ensinar.

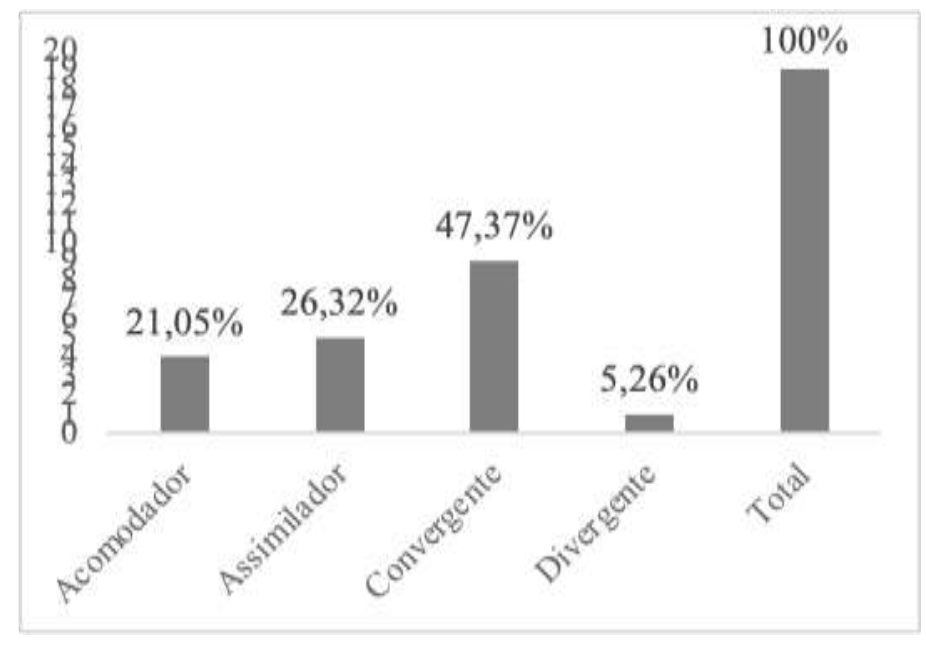

Fonte: Autores (2021).

Os dados encontrados neste trabalho vêm de encontro a pesquisa de Souza et al. (2013), onde estes autores aplicaram o IEA buscando identificar os estilos de ensino de 15 professores do Curso de Administração. Foi verificado que 53,34\% dos professores possuíam o estilo Tutor, ou seja, apresentaram características do estilo de aprendizagem Convergente. Este estilo visa à produtividade dos alunos e costumam combinar aulas em sala, com aulas em laboratórios e atividades extraclasse (Valente, et al., 2007). Ao mesclar estes tipos de atividades para a aquisição e a construção do conhecimento, conforme os estudantes melhor aprenderiam neste estilo, a figura do professor, enquanto tutor, é necessária para auxiliar os estudantes nas aulas, os quais vão em busca de relacionar conceitos e definições (Prado, et al., 2021).

Os professores que se baseiam com estilo de aprendizagem Convergente para ensinar, preferem a aplicação prática de teorias, inclinando-se a lidar com atividades técnicas ou práticas. Kolb $(1999 ; 2015)$ cita exemplos como o experimento de simulações, role-plays ou tarefas desenvolvidas em ambiente do laboratório. Cerqueira (2000) corrobora para esta discussão ao afirmar que, pelo estilo convergente, o aluno tem mais êxito quando lida com fatos que têm uma única solução correta. Nesse caso, usa-se o raciocínio hipotético-dedutivo, sendo que o ponto forte está na aplicação prática das ideias, como também na definição do problema e tomada de decisões (Cerqueira, 2000).

Nota-se que, a partir dos dados da Figura 3 e 4, de modo geral, que os estilos de ensino predominante dos professores (convergente) apresentam compatibilidade com os estilos de aprendizagem prevalentes (assimilador e convergente) dos estudantes. Como salientam Souza et al. (2013), é possível evidenciar compatibilidades entre os estilos de aprendizagem e os métodos de ensino.

Os dados do presente trabalho também foram comparados entre os estudantes (Figura 5) e os professores (Figura 6), pertencentes à Escola 1 (E1) e à Escola 2 (E2). De modo geral, nota-se que o estilo de aprendizagem predominante dos estudantes é o Assimilador. 
Figura 5. Estilo de aprendizagem dos estudantes por escola e ano escolar.

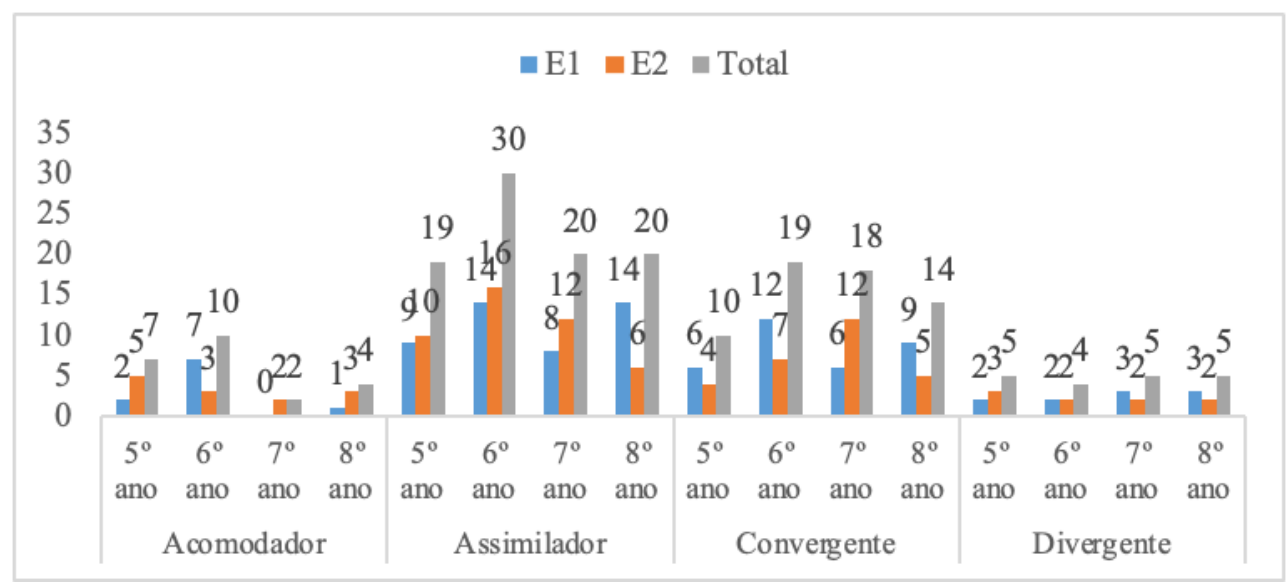

Fonte: Autores (2021).

Entre os resultados observados está para uma distribuição mais uniforme entre os estilos de aprendizagem para o $5^{\circ} \mathrm{e}$ $8^{\circ}$ anos da E2. Esse dado expressa que estes estudantes apresentam diversas preferências nas formas de aprender. Este perfil acaba sendo um desafio para os professores, os quais podem ter muito êxito com a aprendizagem dos estudantes, se eles conseguirem explorar dentro de sua prática pedagógica os quatro estilos de aprendizagem (assimilador, acomodador, convergente e divergente) com os seus alunos. $\mathrm{O} 5^{\circ}, 6^{\circ}$ e $8^{\circ}$ anos da E1 e os $7^{\circ}$ anos apresentam o Assimilador e o Convergente enquanto estilos de aprendizagem predominantes.

Conforme Lima Filho et al. (2016), conhecer o estilo de aprendizagem dos estudantes trata-se de uma excelente oportunidade para que os professores consigam estimular nos seus alunos mecanismos cognitivos que possam colaborar no desenvolvimento de conhecimentos, habilidades e competências para além daquelas relativas ao seu estilo de aprendizagem predominante. Além disso, essa ação possibilita auxiliar o professor a pensar no planejamento de suas atividades, refletindo sobre o perfil dos estudantes e a maneira como se comporta o processo do aprendizado discente (Cerqueira, 2000; 2008; Lima Filho, et al., 2016).

Os dados sobre o estilo de aprendizagem dos professores, que se baseiam para ensinar, de modo geral, concentram-se no estilo Convergente para a E2, e no Assimilador para a E1, mostrando distribuição mais uniforme entre os estilos nesta última escola (Figura 6).

Figura 6. Estilo de aprendizagem que os professores se baseiam para ensinar, por escola.

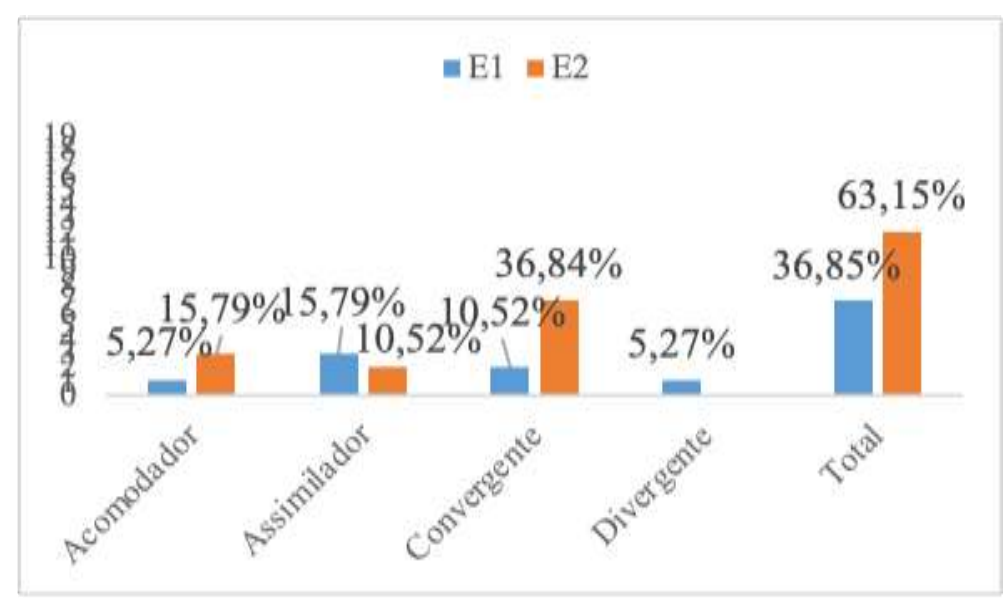

Fonte: Autores (2021). 
Em ambas escolas, nota-se que o perfil dos professores e estudantes são compatíveis quanto aos seus estilos de aprendizagem dominantes. Essa característica é reiterada por Valente et al. (2007), os quais corroboram para essa discussão, afirmando que os resultados da aplicação do IEA podem condizer com a realidade estudada:

Quando da apresentação dos resultados do teste aos sujeitos pesquisados, que ao ler as características do estilo de aprendizagem no qual se inseriam de acordo com o teste Kolb, confirmavam serem aquelas as suas preferências no modo de aprender, demonstrando não somente uma aceitação em relação aos resultados do teste como também, em alguns casos, uma certa admiração com a coincidência das características do seu perfil e de seu estilo de aprendizagem (Valente, et al., 2007, p. 64).

Os achados da pesquisa de Souza et al. (2013) faz pensar sobre o modo como os professores tendem a aprender, o que pode refletir na maneira que eles ensinam. Logo, se os professores apresentam prevalência para um determinado estilo de aprendizagem, há uma possiblidade para que eles repliquem essa preferência de estilo para o modo como eles irão ensinar os seus alunos. O estudo de Meurer et al. (2018) faz refletir a respeito do desempenho dos alunos a partir dos suas preferências de aprendizagens.

Estes mesmos autores verificaram os percentuais de reprovações por alunos, percebendo que somente 5,88\% dos alunos com estilo assimilador reprovaram em alguma matéria, frente a 14,29\% dos divergentes, $16,28 \%$ dos convergentes e $30 \%$ dos acomodadores. Conforme Meurer et al. (2018), estatisticamente, o desempenho dos assimiladores é maior perante os acomodadores. Logo, entende-se que o perfil de aprendizagem pode ser uma das causas desta diferença, pois, segundo o que Meurer et al. (2018) afirmam, os estudantes assimiladores tendem a ter maior facilidade teórica, coincidindo também com o estilo dos professores. No caso deste estudo, esses dados dialogam mais com a realidade da E1, como mostra a Figura 6.

O estilo de aprendizagem Acomodador e Divergente não mostraram predominâncias nos resultados relacionados aos estudantes. Somente o professor P5, da E1, apresentou ser divergente. Este estilo de aprendizagem, segundo Kolb (1999, p. 5), a pessoa tende por "afastar-se das soluções convencionais, e optar por possibilidades alternativas", onde preferem dialogar a partir de discussões, brainstorming (produção de ideias) e trabalhos em grupo. São considerados como criativos, geradores de alternativas, tendo a capacidade de reconhecerem os problemas e compreender as outras pessoas (Cerqueira, 2000; 2008).

$\mathrm{O}$ estilo de aprendizagem Acomodador é comumente conhecido por aqueles que procuram adaptar o aprendido para seu próprio uso, usando a criatividade para mudar e fazer melhor. Os dados referentes ao estilo de aprendizagem Acomodador se remetem, também, ao perfil de uma parcela dos professores. Este estilo é observado em pessoas que gostam de desafios e de novas experiências. Pessoas com esta preferência de aprendizagem se destacam em aulas de campo, projetos, atividades práticas e com jogos (Kolb, 2015; Acrani, et al., 2020). Tirar conclusões a partir do que se experimenta e se vivencia é uma das preferências desse estilo de aprendizagem, onde se usa mais a intuição do que a lógica (Acrani, et al., 2020). Os professores que exploram esse estilo de aprendizagem com seus alunos, envolve-los em atividades mais práticas e, frequentemente, o método utilizado é a tentativa e o erro para resolver problemas, o que nem sempre dá certo (Leite, et al., 2020).

A partir do que foi exposto, concorda-se com Guerra et al. (2020), que a associação dos estilos de aprendizagem ao trabalho do professor pode promover uma comunicação efetiva e um aprendizado mais homogêneo na sala de aula. Isso porque o docente tem a capacidade de percepção de qual o melhor estilo de aprendizagem se adequa a cada aluno. Deste modo, cabe ao professor buscar conhecimento e trazer propostas de atividades que contemplem todas as formas de aprendizados, valorizando, assim, cada aluno em sua particularidade (Guerra, et al., 2020). 


\section{Conclusão}

O objetivo do estudo foi analisar as preferências de estilo de aprendizagem de estudantes e professores do Ensino Fundamental. Os estilos de ensino predominante dos professores (convergente) apresentaram compatibilidade com os estilos de aprendizagem prevalentes dos estudantes (assimilador e convergente). Apesar dos professores apresentarem a predominância do estilo de aprendizagem Convergente, notou-se que o perfil destes partícipes (Pragmático, Teórico e Ativista) abarcam uma distribuição uniforme entre os estilos de aprendizagem nos quais os docentes se baseiam para ensinar. Isso remete o entendimento de que é possível explorar mais estilos de aprendizagem dos estudantes, para além dos expressos neste estudo.

A análise dos dados, quando comparados entre os estudantes, por ano escolar, e os professores, por escola, denotou que há diferentes perfis de estilos de aprendizagem para cada ano $\left(5^{\circ}, 6^{\circ}, 7^{\circ}\right.$ e $8^{\circ}$ anos), bem como há distintos perfis de estilo de ensino em cada escola (E1 e E2). Essas características, relativas às preferências de aprendizagem dos estudantes e as tendências de estilo de ensino dos professores, auxiliam no entendimento dos perfis de aprendizado e de ensino, inclusive, a compreender melhor como as pessoas pensam e organizam as ideias.

Refletindo a respeito da identificação dos estilos de aprendizagem, compreende-se que o IEA de Kolb pode ser uma importante ferramenta para uso de professores e nas escolas, o que traz importantes conhecimentos acerca dos diferentes estilos de aprendizagem dos alunos. Sendo assim, torna-se relevante o uso de ferramentas e instrumentos no ensino que possam ser compatíveis com os estilos de aprendizagem dos estudantes, justamente para se conseguir melhores resultados dentro do processo de ensino e de aprendizagem, de modo a facilitar a aprendizagem dos educandos.

Alguns aspectos limitantes do estudo necessitam ser comentados, na forma de colaborar com o desenvolvimento de trabalhos futuros. Ao se deparar com um perfil de estudantes do Ensino Fundamental, percebeu-se que os alunos do $5^{\circ}$ ano apresentaram maior dificuldade para compreender a proposta do estudo e entender as instruções no momento de responder as questões do IEA de Kolb, isso embora o pesquisador houvesse esclarecido as dúvidas que iam surgindo por parte dos respondentes. Alguns termos, constantes no IEA, como "Eu sou uma pessoa intuitiva", geraram frequentes dúvidas a respeito do significado de "intuitiva". Esse aspecto fez refletir que a versão que o questionário foi aplicado pode sofrer adaptações para que fique mais claro ao público o seu conteúdo, e gerar melhores entendimentos da proposta.

Acredita-se que este estudo diagnóstico ajudou a pensar acerca da adoção do IEA como uma estratégia pedagógica aliada ao ensino. Aliás, deixando reflexionamentos sobre a possiblidade de utilizar esse instrumento combinando com a abordagem de Metodologias ativas. O entendimento da importância dessa combinação é resultante de uma abordagem envolvendo uma aprendizagem ativa, que pode ser produtiva no mapeamento dos estilos de aprendizagem e com foco na escolha de metodologias e estratégias de ensino pelo professor que possam atender a pontos em que os estudantes necessitam a melhorar enquanto aprendizado.

Como a literatura específica tem afirmado, há a possibilidade de utilizar essa ferramenta de investigação como uma estratégia pedagógica que sirva para dar sustentação a uma série de intervenções pedagógicas, favorecendo a escolha de metodologias ativas. Inclusive, alguns estudos relatam sobre a importância de mapear os estilos de aprendizagem dos estudantes antes da realização das intervenções pedagógicas e da escolha de que metodologias serão adotadas pelo professor. Isso porque, no momento em que se escolhe uma abordagem que dê conta de explorar um número maior estilos de aprendizagens diferentes dos educandos, maior seria o êxito e eficácia do uso das Metodologias ativas no processo de ensino e de aprendizagem. A abordagem utilizando as Metodologias ativas tem potencial para otimizar este processo. Os estudos têm pronunciado isso, entendendo que os testes aplicados têm objetivado facilitar a compreensão de como o aluno melhor aprende, como ele lida com as ideias e com situações do dia a dia. 


\section{Agradecimentos}

Ao Instituto Federal de Educação, Ciência e Tecnologia Farroupilha (IFFar) pelo apoio financeiro no desenvolvimento do estudo.

\section{Referências}

Acrani, S., Benze Junior, R. A., Scarambone, B. M., Nicula, B. S., Melo, K., Lopes, L. A., Nogueira, M. B. R., Fernandes, R. D. \& Santos, P. P. B. F. (2020). A utilização do inventário de Kolb como estratégia de diagnóstico e planejamento de metodologias ativas para otimizar o processo de ensino aprendizagem. Brazilian Journal of Development, 6(2), 7882-7893, fev.

Assunção, T. V. \& Nascimento, R. R. (2019). O inventário de estilos de aprendizagem de David Kolb e os professores de ciências e matemática: diálogo sobre o método de ensino. Góndola, Enseñanza y Aprendizaje de las Ciencias, 14(1), 14-34.

Bogdan, R. C. \& Biklen, S. (1994). Investigação qualitativa em educação. Porto, Portugal: Porto Editora.

Brasil. (2018). Ministério da Educação (MEC). Notícias sobre o Saeb. Brasília: MEC, 2018. http://portal.inep.gov.br/.

Cerqueira, T. C. S (2008). Estilos de aprendizagem de Kolb e sua importância na Educação. Revista De Estilos De Aprendizaje, 1(1). http://revistaestilosdeaprendizaje.com/article/view/866.

Cerqueira, T. C. S. (2000). Estilos de aprendizagem em universitários. Tese (Doutorado em Educação) - Faculdade de Educação, Universidade Estadual de Campinas (UNICAMP). Campinas: UNICAMP

Freeman, S., Eddy, S. L., McDonough, M., Smith, M. K., Okoroafor, N., Jordt, H. \& Wenderoth, M. P. (2014). Active Learning Increases Student Performance. Proceedings of the National Academy of Sciences, 111(23), 8410-8415.

Gil, A. C. (2008). Métodos e técnicas de pesquisa social. $6^{\text {a }}$ Ed. São Paulo: Atlas

Guerra, M. J. L., Naidon, T. C. \& Viana, L. A. F. C. (2020). A BNC da formação de professores da educação básica: considerações sobre os estilos de aprendizagem. Research, Society and Development, 9(10), 1-18, e6039109111.

Jesus, E. M. S., Santos, D. V., Vieira, M. L. C. \& Carvalho, A. A. (2017). Metodologias de ensino e os estilos de aprendizagem na graduação em Farmácia: um estudo piloto. RPGE - Revista on-line de Política e Gestão Educacional, 21(1), 621-639, out.

Kolb, D. A. (1984). Experiential learning: Experience as the source of learning and development. New Jersey: Prentice-Hall.

Kolb, D. A. (1999). Inventario de estilos de aprendizaje (IEA). Versión 3. TRB Hay/MacBer

Kolb, D. A. (2015). Experiential Learning: Experience as the Source of Learning and Development. New Jersey: Pearson Education. Second edition.

Leite, E. A. M., Lencastre, J. A., Silva, B. D. \& Neto, H. B. (2020). Estilo de aprendizagem em ambiente virtual: um estudo com professores do ensino fundamental em formação em serviço. Research, Society and Development, 9(7), 1-27, e467973790.

Lima Filho, R., Bezerra, E. \& Silva, T. (2016). Estilo de aprendizagem dos alunos do curso de Ciências Contábeis. Revista Gestão Universitária na América Latina - GUAL, 9(2), 95-112.

Meurer, A. M., Pedersini, D. R., Antonelli, R. A., \& Voese, S. B. (2018). Estilos de aprendizagem e rendimento acadêmico na universidade. REICE: Revista Iberoamericana sobre Calidad, Eficacia y Cambio en Educación, 16(4), 23-43.

Minayo, M. C. S. \& Guerriero, I. C. Z. (2014). Reflexividade como éthos da pesquisa qualitativa. Ciência \& Saúde Coletiva, 19(4), $1103-1112$.

Nogueira, D. R., Espejo, M. M. S. B., Reis, L. G. \& Voese, S. B. (2012). Estilos de aprendizagem e desempenho em educação a distância: um estudo empírico com alunos das disciplinas de contabilidade geral e gerencial. Revista de Educação e Pesquisa em Contabilidade (REPeC), 6(1): 54-72.

Paim, M. G., Goldschmidt, A. I., \& Loreto, E. L. S. (2021). Concepções prévias de alunos do 9o ano do Ensino Fundamental sobre o processo de cicatrização e sua relação com a Biologia Celular. Research, Society and Development, 10(8), e10610817000.

Prado, M. E. B. B., Dias, F. A. S., Bernardo, T. C. S, \& Soares, E. A. (2021). Estilos de Aprendizagem e o Desenvolvimento do Estudante Adulto. Revista de Ensino, Educação e Ciências Humanas, 22(1), 52-55.

Prandina, M., \& Santos, M. (2017). A Educação Física escolar e as principais dificuldades apontadas por professores da área. Horizontes - Revista de Educação, 4(8), 99-114. https://ojs.ufgd.edu.br/index.php/horizontes/article/view/5745.

Santos, E. V. \& Cunha, I. C. K. O. (2013). Estilos de aprendizagem de alunos num curso técnico de enfermagem. In: Anais do I Congresso Online de Gestão, Educação e Promoção da Saúde. São Paulo.

Santos, C. A., Panucci Filho, L. \& Hein, N. (2018). Estudo dos fatores associativos dos estilos de aprendizagem dos acadêmicos do Curso de Ciências Contábeis. Revista Gestão Universitária na América Latina - GUAL, 11(2), 70-88. 
Research, Society and Development, v. 10, n. 12, e252101220503, 2021

(CC BY 4.0) | ISSN 2525-3409 | DOI: http://dx.doi.org/10.33448/rsd-v10i12.2053

Souza, G. H. S., Lima, N. C., Costa, A. C. S., Santos, P. C. F., Junior, J.F.V.P. \& Penedo, A. S.T. (2013). Estilos de aprendizagem dos alunos versus métodos de ensino dos professores do curso de Administração. In: Anais do XXXVII Encontro da Associação Nacional de Pós-Graduação e Pesquisa em Administração. 37, Rio de Janeiro: AnPAD. http://www.anpad.org.br/admin/pdf/2013_EnANPAD_EPQ16.pdf.

Valente, N. T. Z., Abib, D. B. \& Kusnik, L. F. (2007). Análise dos estilos de aprendizagem dos alunos e professores do curso de graduação em ciências contábeis de uma universidade pública do estado do Paraná com a aplicação do inventário de David Kolb. Contabilidade Vista \& Revista, 18(1), 51-74. 